more thorough advance care planning conversations occurring in six patients (10\%). Almost $30 \%$ of patients audited were positive for COVID-19 infection. Where CPR was felt to be of no clinical benefit, COVID-19 infection was rarely (2\% of patients) the named medical condition documented in the DNACPR.

Conclusions Documentation regarding the timing of DNACPR decisions and DNACPR discussions was of a high standard, despite increased pressures during the COVID-19 pandemic. Advance care planning discussions occurred, however, further analysis would be necessary to fully evaluate the quality of these discussions.

\section{P-67 COMMUNITY PALLIATIVE CARE RESPONSE TO SUPPORT CARE HOME RESIDENTS AND STAFF DURING THE COVID-19 PANDEMIC}

Joy Ross, Helen King. St Christopher's Hospice, London, UK

10.1136/spcare-2021-Hospice.85

Aim To review our response to support residents and staff in Bromley Care Homes during the COVID-19 pandemic (April 2020-March 2021), reflect on our learning, joint working with colleagues and identify good practice to inform future models of care.

Methods We collated clinical activity data and key case reviews of care home patients referred to our service over the year. Contemporaneous notes from formal/informal reflections and debriefs (internal and joint with GPs/CCG/other professionals) were reviewed. Themes from feedback of care home staff and managers (ad hoc and formal focus groups) were included with personal reflections.

Results 345 patients were referred from 32 care homes. The majority (45\%) in Quarter 1 (first wave), 14\% in both Q2/Q3 and $27 \%$ in Q4 (second wave). Median age 89(53-110) with $69 \%>85$ years; two-thirds female. $80 \%$ had a non-malignant primary diagnosis. Just over half died within the year; median time referral-to-death $17(0-229)$ days, $81(23 \%)$ remained on the caseload April 2021.

Key themes in Q1 included: limited effectiveness of virtual assessments, atypical patient presentations, significant impact of social isolation on mental health/function, with families unable to advocate and inconsistent messaging about visiting rights. Care home staff were distressed, burnt out, feeling unsupported. In Q2/Q3 regular GSF meetings with care home-GPs, virtual teaching (webinars/ECHO) and staff 'cascade project' study days helped consolidate learning. The second wave was heralded by an outbreak in extra-care housing; care home-GPs were self-isolating. We led urgent senior clinical review and response.

In Q4, daily COVID-19 monitoring meetings were key (representation from CCG, Public Health, Pharmacy, CH-GPs and St Christopher's). Over a month, successive outbreaks were identified and resources coordinated to ensure clear advance care plans, timely review, targeted multiprofessional support to care home staff. Learning from each setting informed changes to approach in the next, including: understanding culture, correcting/enhancing infection control procedures, improved shared decision making and addressing hydration.

Conclusion Senior clinical leadership, cross-boundary flexible working and willingness to learn together were vital.

\section{INTRODUCING A CARE HOME PROJECT TO SUPPORT END-OF-LIFE CARE DURING A PANDEMIC}

Jayne Tooth, Toni Flanagan. St Giles Hospice, Lichfield, UK

10.1136/spcare-2021-Hospice.86

Seventy per cent of care home residents die in a care home (Public Health England, 2017) therefore a large part of what care homes provide is end-of-life care (Social Care Institute for Excellence, 2017). Since April 2020 there were 173,974 deaths of care home residents which was an increase of $19.5 \%$, meaning care homes were dealing with more resident deaths than ever before (Office for National Statistics, 2021). The care home project was introduced to sixteen care homes across a geographical area in April 2020 in response to the COVID-19 pandemic.

The aim of this project was to enable care homes to identify residents who were in the last year of life. This was achieved through weekly support either by phone or video conferencing platforms using an empowerment approach. Once residents were identified the facilitators prompted care home staff to consider the following; Do Not Attempt Cardiopulmonary Resuscitation orders, emergency care plans, anticipatory medications and any communications which may be needed with the GP, resident or resident's family.

To support learning, a root cause analysis was completed following a resident's admission to hospital to determine if the admission was avoidable or unavoidable. A reflective debrief was also conducted following each death. All of these were subjected to thematic analysis. The analysis identified several findings including having rescue packs of antibiotics and clear, concise completed ReSPECT forms could make hospital admissions avoidable. The care home staff were also able to clearly reflect on the impact of the COVID-19 pandemic on the end-of-life care residents received, with the visiting restrictions causing a great deal of distress to both loved ones and care home staff.

Overall, the care home project supported a number of care homes and their staff to provide good quality end-of-life care during unprecedented times using learning from across the project to inform all homes.

\section{P-69 CARE HOME DESIGNATED TO TAKE COVID-19 PATIENTS - PALLIATIVE CARE SUPPORT}

Belinda Pomeroy. Salisbury Hospice, Salisbury, UK

\subsection{6/spcare-2021-Hospice.87}

Background Three Acute NHS Trusts were reaching maximum bed capacity by January 2021 due to the COVID-19 Pandemic. A local recently refurbished care home was identified as a suitable COVID-19 discharge facility for COVID-19 positive and COVID-19 contact patients whose ceiling of care could be managed in this setting (including oxygen therapy) to help ease bed pressures.

Aims

- To support primary care and care home staff with symptom control and decision making for those patients who were end-of-life.

- To help avoid readmission back into the Acute NHS Trusts who were already at capacity.

- To support the relatives of these patients. 
Method From January until the end of March 2021 support was provided to the designated care home in the form of:

- A weekly virtual ward round. Members on the virtual ward round included a GP, the Lead Nurse from the care home, an Advanced Nurse Practitioner, a paramedic and a Palliative Care Nurse Specialist.

- The provision of regular telephone calls throughout the week was dependant on need from the care home.

- If there were any particular patients that the care home staff had concerns about a clinical nurse specialist would be available at weekends and bank holidays for advice and support.

- Telephone calls to relatives.

Results

- Prevention of patients being readmitted to the Acute NHS Trusts following the input from specialist palliative care.

- Quality symptom control provided for patients not for escalation.

- Quality end-of-life care.

- Support for care home staff.

Conclusion The specialist palliative care input made a positive contribution to the care of patients and also to the symptom control of those patients who were end-of-life. Staff felt supported in looking after these patients at a time when care home staff were generally feeling very isolated.

\section{P-70 THE EVOLVING ROLE OF RAPID RESPONSE CLINICAL NURSE SPECIALIST DURING THE COVID-19 PANDEMIC}

Ross Chirgwin. St Helena Hospice, Colchester, Essex

10.1136/spcare-2021-Hospice.88

Background Having access to a community non-medical prescriber (NMP) can enhance patient care by anticipating and responding quickly to symptom control issues at end-of-life and supporting patients to die within their preferred place of care (McGleish, Sivell, Nelson, 2015). With the predicted surge in demand that would overwhelm our local healthcare the end-of-life hub was expanded which included increased NMP capacity.

Aim To meet the predicated demand in services during the COVID-19 pandemic the end-of-life hub would have rapid response clinical nurse specialists (NMPs) available to rapidly assess and provide specialist palliative care advice and symptom control within the community.

Method All of the NMPs within the community team were redeployed to the end-of-life hub. By expanding the team this enabled access to NMPs seven days a week and at the height of the pandemic an NMP was available 24/7 for patients who required rapid assessment. Within the end-of-life hub there was also a quick implementation of access to emergency medications that the NMPs were able to obtain. This enabled the team to provide timely symptom control when accessing medications from a community pharmacy was not available.

Outcome Support was provided to patients who were COVID19 positive or with a life-limiting illness that required rapid assessment either within their own home and critically those within nursing or residential placements. Assessment was often challenging due to limited information being available and therefore detailed assessment was needed to ensure the best outcome for each individual.
Conclusion The NMPs have now been permanently redeployed to the end-of-life hub and continue to provide cover seven days a week. The role has been critical in supporting patients within the community by enabling rapid assessment, symptom management and allowing patients to die within their preferred place of care.

\section{P-71 END-OF-LIFE HUB- COORDINATING END-OF-LIFE CARE DURING COVID-19 PANDEMIC}

Ross Chirgwin, Nicky Coombes. St Helena Hospice, Colchester, UK

10.1136/spcare-2021-Hospice.89

Background With the concern that demand would overwhelm our local healthcare services our hospice in the home team was expanded to develop an end-of-life care hub. The hub would lead and co-ordinate all non-acute hospital end-of-life care within the locality.

Aim The end-of-life hub would ensure the efficient use of the entire end-of-life resources across the locality and enable timely access to care. This involved working in collaboration with external services including primary care, community nursing, ambulance service and local community hospitals.

Method The end-of-life hub is supported by call handlers, senior staff nurses, healthcare assistants and clinical nurse specialists and was operational 24/7. The main functions of the hub included:

- Single point of contact for all end-of-life matters for professionals, patients and family members 24/7.

- Receive diverted call from NHS 111 and out-of-hours services where patients were either COVID-19 positive and not suitable for acute admission or approaching end-of-life.

- Provision of 24/7 medical advice and specialist palliative care prescribing capability.

- Provision of 24/7 rapid response visiting in partnership with community nursing.

- Coordinating flow through all non-acute community hospital admissions for end-of-life care including medical and pharmacy support.

- Coordinating the expanding virtual ward service.

- Liaising with central continuing healthcare team on care that could not be provided through virtual ward.

- Coordinate the electronic end-of-life register (My Care Choices).

Outcome The end-of-life hub received a total of 45,670 calls into the service over the past year. During this time a total of 4943 referrals were received and processed through the endof-life hub which coordinated their care and ongoing support within the locality.

Conclusion The end-of-life care hub continues to develop and remains a core service within the community to continue to increase our reach to serve our local population.

\section{P-72 TURNING THAT FROWN UPSIDE DOWN; THROUGH COVID-19 AND BEYOND}

Cathy Hunt. Garden House Hospice Care, Letchworth, UK

10.1136/spcare-2021-Hospice.90 